\title{
Técnicas para análise de risco: uma avaliação da literatura sobre gerenciamento de projetos de SI
}

\author{
Edmir Parada Vasques Prado \\ Doutor em Administração pela Universidade de São Paulo (USP). Professor Doutor MS-3 da USP. \\ E-mail: eprado@usp.br (Brasil)
}

\author{
Paulo Mannini \\ Mestre em Sistemas de Informação pela Universidade de São Paulo (USP). \\ E-mail: paulomannini@gmail.com (Brasil)
}

\section{RESUMO}

Este artigo trata de técnicas de gerenciamento de risco em projetos de sistemas de informação (SI). Dentro desse contexto, esta pesquisa tem como objetivo identificar as técnicas descritas na literatura para o gerenciamento de riscos em projetos de SI. A fundamentação teórica da pesquisa foi baseada na literatura sobre gerenciamento de projetos consultada nas bases de dados da Scopus, IEEE e Science Direct. Trata-se de uma pesquisa qualitativa, que contou com uma amostra inicial de 227 artigos, dos quais foram extraídos 25. Os dados foram coletados no primeiro semestre de 2017 e entre os resultados obtidos destacam-se: 1) técnicas usadas para entrada de dados em projetos de SI seguem aquelas utilizadas em projetos de outras áreas; 2) nas técnicas usadas para tratamento de dados se destacam as que utilizam a técnica Fuzzy, sendo que simulações e técnicas baseadas primordialmente em probabilidade são pouco usadas; e 3) as técnicas para apresentação de dados utilizam principalmente listas de priorização.

Palavras-chave: Análise de risco. Gerenciamento de projetos. Revisão sistemática da literatura. 


\title{
Risk analysis techniques: an evaluation of the literature about information system project management
}

\begin{abstract}
This article addresses risk management techniques in information system (IS) projects. Within this context, this research aims to identify the techniques described in the literature about risk management in IS projects. The research reference framework was based on the literature about project management within the SCOPUS, IEE and Science Direct databases. This is a qualitative research, which had an initial sample of 227 articles, from which 25 were extracted. Data were collected in the first semester of 2017 and among the results, we could find: (1) techniques used for data entry in IS projects follow those used in other fields; (2) Fuzzy was the most used technique for risk analysis, however simulations and techniques based primarily on probability are little used; and (3) data presentation techniques mainly use prioritization lists.
\end{abstract}

Keywords: Risk analysis. Project management. Systematic review of literature.

\section{Como referenciar em APA:}

Prado, E. P. V., \& Mannini, P. (2018). Técnicas para análise de risco: uma avaliação da literatura sobre gerenciamento de projetos de si. R.G.Secr.,GESEC, 9(3).

\section{Como referenciar em ABNT:}

PRADO, E. P. V.; MANNINI, P. (2018). Técnicas para análise de risco: uma avaliação da literatura sobre gerenciamento de projetos de si. R.G.Secr.,GESEC, v. 9, n. 3, Dez. 2018.
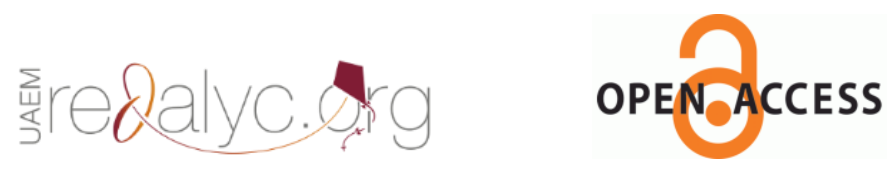


\section{Introdução}

Investir na análise de riscos como parte do gerenciamento de projetos é uma prática importante para diminuir o potencial de falha em projetos. Porém, para permitir que o gerenciamento seja feito de forma adequada, existem modelos de referência disponíveis na literatura. Esses modelos podem ser utilizados por profissionais envolvidos no gerenciamento de projetos com o intuito de facilitar o alcance dos objetivos do projeto. De acordo com Patah e Carvalho (2012), existem diversos conjuntos de modelos de gerenciamento de projetos, sendo que os mais difundidos em organizações brasileiras são: o conjunto de boas práticas denominado Project Management Body of Knowledge (PMBoK) disponibilizado pelo Project Management Institute (PMI, 2017); o método baseado em processos de gerenciamento de projetos denominado Projects In Controlled Environments (PRINCE2) disponibilizado pelo Office of Government Commerce (OGC, 2009); e o referencial de competências em gestão de projetos denominado IPMA Individual Competence Baseline (ICB) disponibilizado pelo International Project Management Association (IPMA, 2015).

Apesar da existência de uma extensa literatura sobre gerenciamento de projetos, os resultados obtidos com os projetos não têm sido bons. Segundo o Standish Group (2016), baseado em resultados de grandes projetos de sistemas de informação (SI) apenas $29 \%$ dos projetos são finalizados com prazo, custo e implantação satisfatória. Esta análise também mostrou que $52 \%$ dos projetos foram considerados desafiadores por terem extrapolado o orçamento, ultrapassando prazos planejados ou não obtiveram uma implantação satisfatória. Um destaque para esta análise é que, infelizmente, os projetos que representam os $42 \%$ restantes da análise foram considerados como falhos, pois foram cancelados ou finalizaram sem a utilização do sistema após a implantação.

Uma das explicações para a alta taxa de falhas nesses projetos é a de que os gerentes não tomam as medidas necessárias para analisar apropriadamente os riscos envolvidos (Schmidt, Lyytinen \& Keil, 2001). Por outro lado, segundo Globerson e Zwikael (2002), os gerentes de projeto possuem uma lacuna de conhecimento em relação aos métodos formais para o planejamento do gerenciamento de riscos em projetos. Dentro desse contexto, esta pesquisa busca responder a seguinte pergunta: quais são as técnicas descritas na literatura para o gerenciamento de riscos em projetos de SI? 
A partir da pergunta de pesquisa se definiu como objetivo geral da pesquisa identificar as técnicas descritas na literatura para o gerenciamento de riscos em projetos de SI. Para atingir esse objetivo geral foram definidos três objetivos específicos: 1) identificar, por meio de uma revisão sistemática da literatura, métodos de análise de risco em projetos; 2) analisar as técnicas utilizadas nos métodos identificados na literatura; e 3) classificar por ordem de frequência as técnicas mais citadas na literatura.

No que se refere à estrutura do artigo, primeiramente, foi apresentado uma revisão da literatura sobre os métodos para análise de riscos em projetos. Em seguida é descrito o método empregado na pesquisa, formando a base para a apresentação e análise dos resultados. À guisa de conclusão, a última seção encerra o artigo com o apontamento das conclusões e considerações finais

\section{Métodos para Análise de Riscos em Projetos}

Existem diversos métodos de análise de riscos em projetos descritos na literatura que são utilizados em diferentes contextos e em diferentes áreas. Nesta pesquisa, como forma de identificar e descrever esses métodos, foi utilizada a técnica de revisão sistemática da literatura (RSL). A RSL possui o objetivo de identificar, avaliar e interpretar todo o conteúdo relevante para uma questão de pesquisa, área ou fenômeno de interesse (Kitchenham, Brereton, Budgen, Turner, Bailey \& Linkman, 2009). Segundo esses autores, a RSL possui três etapas principais: 1) planejamento, na qual são identificadas as questões de pesquisa e elaborado o protocolo de revisão; 2) condução, na qual são selecionados os estudos seguindo o protocolo especificado; e 3) relatório, na qual são sumarizados os dados e analisados os resultados.

Por último, cabe definir a diferença entre métodos e técnicas. Para o contexto desta pesquisa foram utilizadas as seguintes definições:

1) Método. É um conjunto das atividades sistemáticas e racionais, executadas em etapas e que permite alcançar o objetivo (Cervo, Bervian \& Silva, 2007; Lakatos \& Marconi, 2010).

2) Técnica. Refere-se a procedimentos práticos que são adotados para realizar um determinado trabalho (Miranda Neto, 2005). São componentes de um método. 


\section{Planejamento da revisão sistemática da literatura}

A questão de pesquisa definida para guiar a seleção de trabalhos relevantes foi: quais métodos estão sendo utilizados para análise de riscos em projetos? A seleção de trabalhos foi feita em bases de dados contendo periódicos relacionados ao gerenciamento de projetos e contendo áreas de conhecimento e assuntos relacionados ao objetivo da pesquisa. As bases de dados utilizadas estão relacionadas a seguir.

1) Scopus (http://www.scopus.com). Reúne informações de 23.000 títulos de revistas de mais de 5.000 editoras de todo o mundo. Abrange as três áreas de conhecimento: Biológicas, Exatas, Humanas. Entre os diversos assuntos abordados, inclui Engenharia, Ciências Sociais e a área multidisciplinar.

2) Science Direct (www.sciencedirect.com). Possui aproximadamente 26.000 livros e 2.200 periódicos. Abrange as três áreas de conhecimento: Biológicas, Exatas, Humanas. Entre os diversos assuntos abordados, inclui Ciências Exatas, Ciências Sociais e a área Multidisciplinar.

3) IEEE (http://ieeexplore.ieee.org). Possui publicações periódicas, normas técnicas e anais de congressos e conferências publicados pelo Institute of Electrical and Electronic Engineers (IEEE) e pela Institution of Engineering and Technology (IET). Abrange a área de Exatas e entre os diversos assuntos abordados, inclui as diversas modalidades de engenharia.

4) ACM DL(https://dl.acm.org). Uma biblioteca de pesquisa que contém trabalhos de periódicos, conferências, revistas técnicas, boletins e livros de todas as publicações da Association for Computing Machinery (ACM).

O período de tempo considerado foi entre 2012 e maio de 2017, visto que se desejava obter os trabalhos mais atualizados sobre o tema. Foram utilizados apenas strings de busca compostas pelas palavras-chave relacionadas à questão da pesquisa e que especificavam o assunto procurado, considerando junções AND e OR e apenas na língua inglesa. O string de busca utilizado foi: "Project Risk Analysis" OR "Project Risk Assessment" AND "Project Risk Management". As quantidades de artigos resultantes das pesquisas nas bases de dados estão disponíveis na Tabela 1, em conjunto os detalhes das consultas realizadas.

Tabela 1 - Artigos por base de dados.

\begin{tabular}{|l|l|r|}
\hline Base de dados & Tipo de busca & $\mathbf{N}^{\mathbf{0}}$ de artigos \\
\hline Scopus & Avançada & 180 \\
\hline
\end{tabular}




\begin{tabular}{|l|l|r|}
\hline Science Direct & Simples & 43 \\
\hline IEEE & Avançada por metadados & 4 \\
\hline ACM & Simples & 0 \\
\hline
\end{tabular}

Fonte: Elaborada pelos autores.

O protocolo considerou os seguintes critérios para seleção dos artigos:

1) Critérios de inclusão. Foram considerados estudos que apresentavam a avaliação ou aplicação de um método de análise de riscos em projetos, seja ele qualitativo ou quantitativo.

2) Critérios de exclusão. Com o objetivo de selecionar apenas trabalhos relevantes, foram considerados os seguintes critérios de exclusão:

- trabalhos que não tratam de gerenciamento de riscos em projetos;

-trabalhos que não abordam os processos de análise qualitativa ou quantitativa de riscos;

- trabalhos que apresentem somente uma revisão da literatura;

- trabalhos que não fornecem o nome do método de análise utilizado;

- trabalhos duplicados nas bases de dados.

- Trabalhos sem o texto completo disponível para consulta.

3) Critério de qualidade. Foram avaliados os detalhes do processo de execução dos métodos de análise de riscos apresentados nos artigos para verificar se o método estava descrito de forma completa e se era possível reproduzi-lo.

\section{Condução da revisão sistemática da literatura}

Para seleção dos trabalhos foram realizados três passos. O primeiro foi a leitura dos títulos e abstracts para verificar se atendiam aos critérios definidos. Em seguida, fez-se a leitura do texto para verificar a adequação do artigo aos objetivos da pesquisa e, por último, analisaram-se os critérios de qualidade definidos. A Figura 1 apresenta a quantidade de trabalhos incialmente identificados nas bases de dados e a quantidade de artigos selecionados após a aplicação dos critérios. 
Figura 1 - Visão geral da seleção de artigos pela RSL.

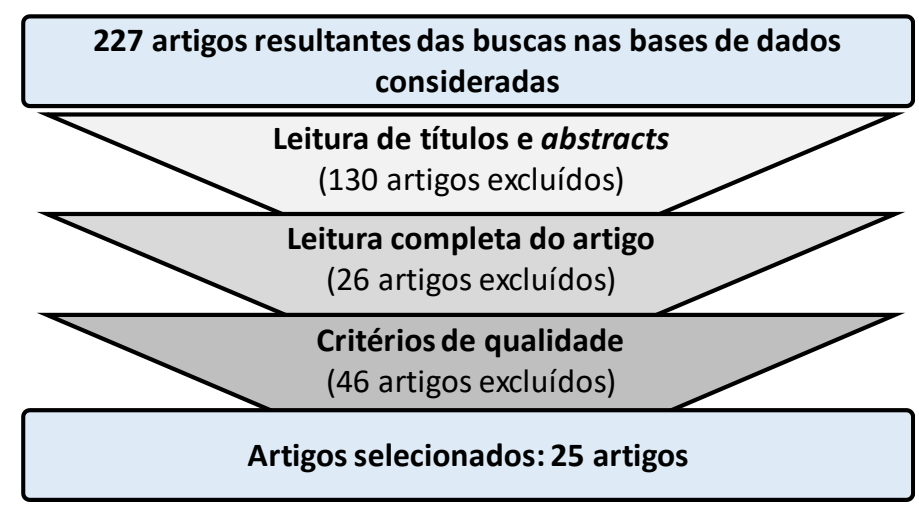

\section{Relatório da revisão sistemática da literatura}

Por meio da RSL realizada foram identificados 17 diferentes métodos, que estão apresentados na Tabela 2. A identificação desses métodos foi feita por meio de análise semântica dos 25 artigos selecionados na RSL (Bardin, 2011). A seguir são descritos cada um desses métodos.

Tabela 2 - Métodos de análise de riscos e trabalhos relacionados.

\begin{tabular}{|c|c|c|}
\hline Código & Método de análise de riscos & Trabalhos relacionados \\
\hline M1 & Analytic Hierarchy Process & $\begin{array}{l}\text { - Marle, Vidal e Bocquet (2013) } \\
\text { - Subramanyan, Sawant e Bhatt (2012) }\end{array}$ \\
\hline M2 & Bayesian Network & $\begin{array}{l}\text { - Hu et al., (2013) } \\
\text { - Odimabo e Oduoza (2014) } \\
\text { - Raoofpanah e Hassanlou (2013) } \\
\text { - Yet et al. (2016) }\end{array}$ \\
\hline M3 & Colored Petri Nets & - Aloini, Dulmin e Mininno (2012a) \\
\hline M4 & Dynamic Bayesian Network & - Yet et al. (2016) \\
\hline M5 & Fuzzy Analytic Hierarchy Process & - Taylan et al. (2014) \\
\hline M6 & Fuzzy Analytic Network Process & - Zegordi, Nik e Nazari (2012) \\
\hline M7 & Fuzzy Expert COCOMO & - Manalif et al. (2012) \\
\hline M8 & Failure Mode and Effect Analysis & - Mohammadi e Tavakolan (2013) \\
\hline M9 & Fuzzy Multiple Criteria Decision Making & - Marmier, Gourc e Laarz (2013) \\
\hline M10 & Fuzzy TOPSIS & - Taylan et al. (2014) \\
\hline M11 & Importance-performance Analysis & • López e Salmeron (2012) \\
\hline M122 & Integrated Index & - Valitov e Sirazetdinova (2014) \\
\hline M13 & Interpretive Structural Modeling & - Aloini, Dulmin e Mininno (2012b) \\
\hline M14 & Matrix-based risk propagation model & - Fang e Marle (2013) \\
\hline M15 & Monte Carlo & $\begin{array}{l}\text { - Arashpour et al. (2016) } \\
\text { - Ayala-Cruz (2016) }\end{array}$ \\
\hline M16 & Relative Importance Index & - Hossen, Kang e Kim (2015) \\
\hline M17 & Three Scenario Approach & - Purnus e Bodea (2013) \\
\hline
\end{tabular}

Fonte: Elaborada pelos autores. 
M1 - Analytic Hierarchy Process (AHP). O AHP é um método baseado em comparação por pares, utilizado para elaborar uma escala de comparação entre diversos fatores analisados (Marle, Vidal \& Bocquet, 2013). Segundo Hossen, Kang e Kim (2015), trata-se de um dos métodos mais populares e poderosos de tomada de decisão. O ponto crucial do AHP é permitir que um tomador de decisão estruture visualmente, sob uma forma hierárquica, um problema envolvido com múltiplos atributos. Zegordi, Nik e Nazari (2012).

M2 - Bayesian Network. Também conhecido como Bayesian Belief Networks, trata-se de um método para representação do conhecimento e reflexão sobre as condições de incerteza, que tem se tornado popular para modelagem de domínios complexos envolvidos com conhecimento e dados incertos (Raoofpanah \& Hassanlou, 2013; Odimabo \& Oduoza, 2014). Segundo Hu et al. (2013) e Yet et al. (2016), esse método tem sido explorado em diversas práticas de gerenciamento de riscos, inclusive no gerenciamento de riscos em projetos de desenvolvimento de software.

M3 - Colored Petri Nets (CPN). O Petri Net é um método gráfico utilizado para especificação, analise e desenho de sistemas de eventos discretos (Aloini, Dulmin \& Mininno, 2012a). Apesar de esses autores obterem sucesso em aplicações de problemas de diversos domínios, algumas desvantagens restringem a aplicação em sistemas grandes e complexos. Com o objetivo de compensar essas desvantagens, surgiu o Colored Petri Net, que é uma extensão da forma tradicional.

M4 - Dynamic Bayesian Network. Diferente do método tradicional, o método Dynamic Bayesian Network utiliza a incerteza e variabilidade dos fatores de riscos em conjunto com fatores econômicos, de modo a realizar previsões em diferentes estágios do projeto (Yet et al., 2016). Para isso, o método considera a realização de estimativas do risco calculadas ao longo do tempo comparadas com a estimativa inicial, ajustando-se os custos em função da previsão inicial e das mudanças identificadas nas estimativas dos riscos.

M5 - Fuzzy Analytic Hierarchy Process. Esse método é uma extensão do método AHP tradicional e é utilizado para solucionar problemas com julgamentos subjetivos e em termos linguísticos dos objetos analisados pelos especialistas (Taylan et al., 2014). Subramanyan, Sawant e Bhatt (2012) e Taylan et al. (2014) utilizaram este método para determinar os pesos entre os fatores analisados utilizando variáveis linguísticas Fuzzy. Em ambos os estudos os autores buscaram analisar riscos em projetos de construção civil e dados obtidos por meio de julgamento dos especialistas da área. 
M6 - Fuzzy Analytic Network Process. Diferente do AHP que é utilizado para solução do problema de independência entre as alternativas ou critérios, o Analytic Network Process (ANP) tende a solucionar o problema de dependência entre essas alternativas ou critérios. $\mathrm{O}$ ANP representa uma generalização do AHP, pois permite uma complexa interrelação entre os atributos de cada camada (Zegordi, Nik \& Nazari, 2012). A inserção do Fuzzy no método ANP permitiu algumas vantagens em relação à melhoria na modelagem da ambiguidade, menor exigência cognitivamente dos especialistas e melhor reflexão dos tomadores de decisão sobre a atitude para lidar com o risco e o grau de confiança sobre a subjetividade das avaliações.

M7 - Fuzzy Expert COCOMO. Trata-se de uma eficiente abordagem para o gerenciamento de riscos em projetos de software, que utiliza conhecimento e expertise obtidos de estimativas de esforço já realizadas previamente para analisar riscos em novos projetos de software (Manalif et al., 2012). Esse autor propôs esse método, que combina a vantagem da técnica Fuzzy com a técnica Expert COCOMO.

M8 - Fuzzy Failure Mode and Effect Analysis. Trata-se de um método para identificação de todos os modos de falha dentro de um sistema, identificando as causas raiz, avaliando os impactos das falhas e planejando ações corretivas. No método tradicional, as prioridades de riscos são baseadas no Risk Priority Number - que pode variar de 1 a 1000 e são obtidos por meio da multiplicação da probabilidade de ocorrência pela severidade da detecção do risco - e podem variar de 1 a 10 (Mohammadi \& Tavakolan, 2013). Segundo os autores, o Fuzzy, quando utilizado junto ao método FMEA para realização de análise de riscos, serve para endereçar as limitações do método FMEA tradicional, tornando-o em um mecanismo simples e efetivo na análise de problemas.

M9 - Fuzzy Multiple Criteria Decision Making. A lógica Fuzzy foi criada para servir como uma alternativa para lidar com a incerteza, imprecisão e problemas complexos difíceis de se resolver quantitativamente. Para realização de análises de riscos em projetos, de acordo com Marmier, Gourc e Laarz (2013), a lógica Fuzzy melhora a precisão das estimativas, aumentando a probabilidade de sucessos dos projetos por meio de um gerenciamento de riscos eficaz.

M10 - Fuzzy TOPSIS. Trata-se de um método de tomada de decisão multicritério que se baseia no conceito de seleção de alternativas que tenham a menor distancia da solução positiva ideal e a maior distância de solução negativa ideal (Taylan et al., 2014). Geralmente 
o método utiliza os dados gerados pela aplicação de outro método, como por exemplo o método Fuzzy AHP ou o método Fuzzy ANP, para estabelecer um ranking de riscos utilizando os pesos obtidos a partir dos outros métodos.

M11 - Importance-performance Analysis. O método combina a medida de importância de cada fator (que são os riscos no caso da utilização para analisar riscos) e sua performance através de uma matriz de duas dimensões (López \& Salmeron, 2012). Segundo esses autores, a partir das informações obtidas com a aplicação do método, é possível prover informações úteis e facilmente interpretadas sobre quais riscos deveriam ser priorizados em função da probabilidade e impacto.

M12 - Integrated Index. O método foi desenvolvido por Valitov e Sirazetdinova (2014) para considerar a natureza híbrida dos riscos, probabilidades, consequências e controle dos riscos. Esse método utiliza os seguintes indicadores para realização do cálculo: probabilidade de ocorrência de um evento; resultados financeiros de um evento de risco; e capacidade de influência na realização do evento de risco ou de suas consequências. Segundo os autores, o método é efetivo em conectar o gerenciamento de riscos com os riscos de maior impacto e probabilidade.

M13 - Interpretive Structural Modeling. Trata-se de um método suportado por computador e baseado em processos Structural Modeling para construir e compreender os fundamentos das relações entre variáveis em um sistema complexo (Aloini, Dulmin \& Mininno, 2012b). O método provê uma forma estruturada para interpretação de um grupo de julgamentos sobre quando e como essas variáveis estão relacionados no sistema. Os processos Structural Modeling geralmente operam como processos de aprendizagem interpretativa de um grupo de indivíduos sobre um sistema. Por meio da contribuição de diversos participantes com dados, ideias, conhecimento e habilidades relacionadas ao sistema, os processos de Structural Modeling oferecem uma melhoria de entendimento sobre esse sistema.

M14 - Matrix-based Risk Propagation Model. O método foi introduzido por Fang e Marle (2013), baseando-se no método Design Structure Matrix (DSM). Usando o DSM, Fang e Marle (2013) criaram uma estrutura matricial binária denominada Risk Structure Matrix, identificando se há ou não interação entre riscos identificados por meio dos julgamentos de especialistas. Posteriormente, além da avaliação do impacto e da probabilidade de cada risco, são avaliadas também as forças entre as interações dos riscos, considerando a probabilidade do risco em ser acionado pela ocorrência de outro risco. 
M15 - Simulação de Monte Carlo. Segundo Arashpour et al. (2016), a simulação de Monte Carlo é um dos métodos mais sofisticados para realização de análise de riscos, utilizado para uma grande variedade de problemas de risco, tais como atrasos no cronograma e extrapolação de custos. Ele é baseado em teorias cientificas que permitem entender e comprovar os riscos considerados de maior impacto nos objetivos principais do projeto (Ayala-Cruz, 2016).

M16 - Relative Importance Index. O trabalho de Hossen, Kang e Kim (2015) utiliza a combinação desse método com os métodos AHP. O AHP é usado para avaliar os impactos e esse método é usado para avaliar as probabilidades de ocorrência de fatores riscos. O Relative Importance Index é um método estatístico para determinação de um ranking de diferentes fatores baseado na probabilidade de ocorrência fornecidas por especialistas.

M17 - Three Scenario Approach. Segundo Purnus e Bodea (2013), trata-se de um método semiprobabilístico. Para aplicação desse método são realizadas três estimativas (otimista, mais provável e pessimista), considerando-se os eventos de risco e os dados iniciais do projeto, tais como duração, volume de trabalho, produtividade, cronogramas e recursos. A partir destas estimativas, as curvas de probabilidade são geradas para prazos, custos e recursos necessários para o projeto. Segundo Liberzon e Archibald (2003), tais estimativas são utilizadas na análise de riscos para definir as probabilidades desejáveis para se alcançar as datas, custos e taxa de utilização de recursos alvo do projeto.

\section{Método da Pesquisa}

Esta pesquisa é fundamentada em uma revisão bibliográfica e apresenta características qualitativas e quantitativas. Nesta seção são apresentados o tipo de pesquisa, as fases da pesquisa, bem como os procedimentos de coleta e tratamento dos dados.

\section{Tipo de pesquisa}

A pesquisa proposta neste trabalho caracteriza-se por ser um estudo bibliográfico e exploratório. Segundo Cervo Bervian e Silva (2007), a pesquisa exploratória visa oferecer informações sobre o objeto de estudo e orientar a formulação de hipóteses para futuras 
pesquisas. Dessa forma, esta pesquisa explora as bases de dados científicas para identificar técnicas de análise de riscos em projetos, que possam servir de base para futuras pesquisas descritivas e explicativas e que possam ser usadas na prática gerencial. Por fim, cabe destacar que este estudo se trata de uma pesquisa quantitativa e transversal do tipo único, pois a coleta de informação foi feita uma única vez.

\section{Fases da pesquisa}

Esta pesquisa foi desenvolvida em quatro fases como ilustra a Figura 2. A primeira fase constituiu a revisão sistemática da literatura apresentada na Seção de Métodos para Análise de Riscos em Projetos. Esse levantamento foi realizado nas bases de dados da IEEE, Scopus e Science Direct e selecionou 25 artigos.

Figura 2 - Fases da pesquisa

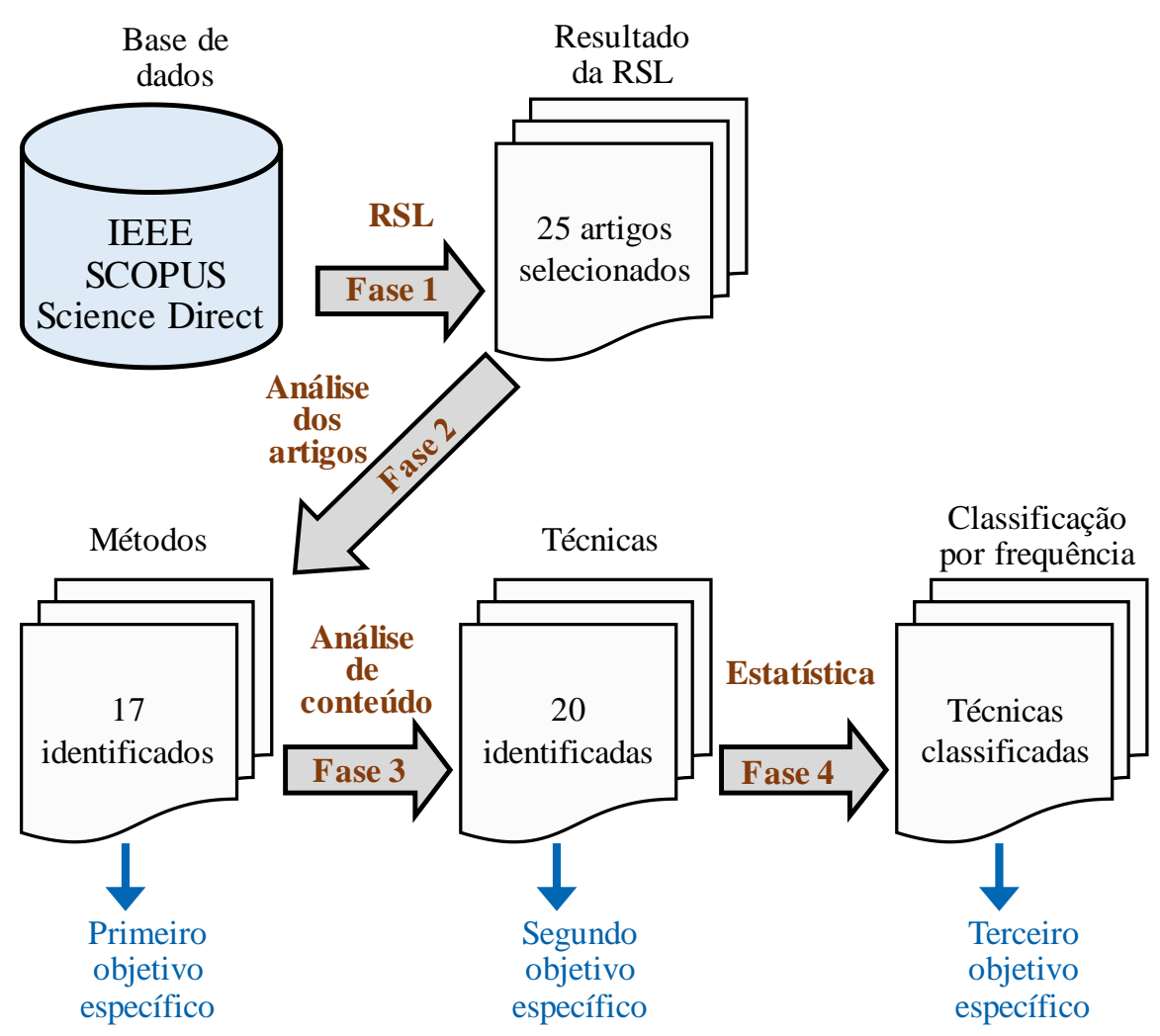


$\mathrm{Na}$ segunda fase foi realizada uma análise nos 25 artigos selecionados pela RSL e identificou 17 métodos utilizados na análise de risco em projetos. Os resultados dessa fase também estão apresentados na Seção de Métodos para Análise de Riscos em Projetos.

$\mathrm{Na}$ terceira fase da pesquisa foi feita uma análise de conteúdo (Bardin, 2011) que identificou 20 técnicas presentes nos 17 métodos de análise de risco identificados na segunda fase. Os resultados desta fase estão apresentados na seção Análise e Resultados.

$\mathrm{Na}$ quarta e última fase foram utilizadas técnicas estatísticas para classificar por frequência de citação na literatura as técnicas identificadas na terceira fase. Os resultados desta fase também estão apresentados na seção Análise e Resultados.

\section{Coleta e análise dos dados}

As unidades de análise desta pesquisa são os métodos de análise de risco identificados na literatura. Os dados foram coletados das bases científicas no primeiro semestre de 2017. Os dados foram analisados inicialmente com técnicas qualitativas e em seguida com técnicas quantitativas cujas características estão descritas a seguir.

1) Análise qualitativa. Esta análise foi feita por meio da aplicação de técnicas de análise de conteúdo (Bardin, 2011). Entre essas técnicas, foi utilizada a análise semântica, que consiste em analisar o sentido das palavras encontradas nos textos. O uso dessa técnica apresenta limitações, entre as se quais destaca a subjetividade presente na análise feita pelos pesquisadores.

2) Análise quantitativa. Esta análise foi feita com uso de técnicas estatísticas descritivas. Entre as limitações do uso desta técnica, destaca-se a impossibilidade de generalizar os resultados, uma vez que outras bases de dados não foram consultadas e a amostra obtida não é significativa da população de artigos científicos sobre análise de risco em projetos.

\section{Análise e Resultados}

A análise dos dados e os resultados estão apresentados em dois tópicos. O primeiro apresenta as técnicas identificadas na literatura e o segundo classifica por ordem de frequência essas mesmas técnicas. 


\section{Técnicas identificadas}

A partir dos 17 métodos identificados na RSL buscou-se, por meio da análise semântica desses métodos, identificar as técnicas utilizadas por eles. Foram identificadas 20 técnicas, as quais estão apresentadas na Figura 3.

Figura 3 - Técnicas usadas pelos métodos.

\begin{tabular}{|c|c|c|c|c|c|c|c|c|c|c|c|c|c|c|c|c|c|c|c|}
\hline \multicolumn{2}{|l|}{ Técnicas } & \multicolumn{18}{|c|}{ Métodos } \\
\hline \multirow[t]{2}{*}{ Descrição } & \multirow[t]{2}{*}{ Frequência } & \multicolumn{6}{|c|}{ Alto } & \multicolumn{5}{|c|}{ Médio } & \multicolumn{7}{|c|}{ Baixo } \\
\hline & & M1 & $\begin{array}{lll}\text { M2 } & \end{array}$ & M5 & M8 & M9 & M15 & M3 & M6 & M10 & M13 & M17 & $\mathbf{M}$ & \begin{tabular}{l|l}
77 & $\mathrm{~N}$
\end{tabular} & M4 & M11 & M12 & M14 & M16 \\
\hline \multicolumn{20}{|l|}{ Entrada de dados } \\
\hline Opinião especializada & 11 & $\bullet$ & $\bullet$ & $\bullet$ & $\bullet$ & $\bullet$ & $\bullet$ & & $\bullet$ & $\bullet$ & $\bullet$ & $\bullet$ & & & & $\bullet$ & & & \\
\hline Bases históricas & 6 & $\bullet$ & $\bullet$ & & & & $\bullet$ & & & & & $\bullet$ & 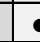 & 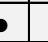 & $\bullet$ & & & & \\
\hline Entrevista & 6 & & & $\bullet$ & & $\bullet$ & $\bullet$ & & $\bullet$ & $\bullet$ & & & & & & & $\bullet$ & & \\
\hline Riscos categorizados por fonte & 5 & $\bullet$ & & $\bullet$ & $\bullet$ & $\bullet$ & & & & & & & 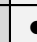 & 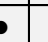 & & & & & \\
\hline Questionário & 3 & $\bullet$ & $\bullet$ & & $\bullet$ & & & & & & & & & & & & & & \\
\hline Delphi & 2 & & & & & & & $\bullet$ & & & $\bullet$ & & & & & & & & \\
\hline Brainstorming & 1 & & & & & & & & & & & $\bullet$ & & & & & & & \\
\hline Observações experimentais & 1 & & $\bullet$ & & & & & & & & & & & & & & & & \\
\hline Riscos categorizados por fase & 1 & $\bullet$ & & & & & & & & & & & & & & & & & \\
\hline \multicolumn{20}{|l|}{ Tratamento de dados } \\
\hline Fuzzy & 7 & $\bullet$ & & $\bullet$ & $\bullet$ & $\bullet$ & & & $\bullet$ & $\bullet$ & & & 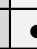 & $\bullet$ & & & & & \\
\hline Interdependência de riscos & 7 & $\bullet$ & $\bullet$ & & & & & $\bullet$ & & & $\bullet$ & $\bullet$ & 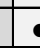 & $\bullet$ & & $\bullet$ & & & \\
\hline Multicritério & 6 & $\bullet$ & & $\bullet$ & $\bullet$ & $\bullet$ & & & $\bullet$ & $\bullet$ & & & & & & & & & \\
\hline Probabilidade e impacto & 5 & $\bullet$ & & $\bullet$ & $\bullet$ & $\bullet$ & & & & & & & & & & & $\bullet$ & & \\
\hline Distribuição probabilística & 4 & $\bullet$ & $\bullet$ & & & & $\bullet$ & & & & & & & & $\bullet$ & & & & \\
\hline Simulação & 4 & $\bullet$ & $\bullet$ & & & & $\bullet$ & $\bullet$ & & & & & & & & & & & \\
\hline \multicolumn{20}{|l|}{ Apresentação de dados } \\
\hline Lista priorizada de riscos & 8 & $\bullet$ & & $\bullet$ & $\bullet$ & $\bullet$ & $\bullet$ & & $\bullet$ & $\bullet$ & $\bullet$ & & & & & & & & \\
\hline Rede de relacionamento & 5 & $\bullet$ & $\bullet$ & & & & & $\bullet$ & & & & $\bullet$ & & & $\bullet$ & & & & \\
\hline Matriz probabilidade-impacto & 5 & $\bullet$ & & & & & & & & & $\bullet$ & & & & & $\bullet$ & $\bullet$ & $\bullet$ & \\
\hline Tabela & 4 & $\bullet$ & $\bullet$ & & & & & $\bullet$ & & & & & & & & & & & $\bullet$ \\
\hline Gráfico & 1 & $\begin{array}{ll}\bullet \\
\end{array}$ & & & & & & & & & & & & & & & & & \\
\hline Total & 92 & \begin{tabular}{|l|}
16 \\
\end{tabular} & 9 & 7 & 7 & 7 & 6 & 5 & 5 & 5 & 5 & 5 & 5 & 4 & 3 & 3 & 3 & 1 & \\
\hline
\end{tabular}

Nota: os métodos de M1 a M17 estão descritos na Tabela 1.

Em relação à entrada de dados nos processos de avaliação de risco de projetos de SI, pelo menos três técnicas usadas em gerenciamento de projetos não foram citadas na literatura de SI: reunião, técnica de grupo nominal e riscos categorizados por área.

Os métodos foram classificados em três grupos, em função do número de técnicas tradicionais utilizadas, usando a estatística de percentil. O grupo com maior número de técnicas apresentou seis métodos: AHP (M1); Bayesian Network (M2); Fuzzy Analytic Hierarchy Process (M5); Failure Mode and Effect Analysis (M8); Fuzzy Multiple Criteria Decision Making (M9); e Monte Carlo (M15). Três desses métodos, usados em projetos de SI, 
são tradicionais na área de projetos como um todo (M1, M2 e M15) e dois métodos (M5 e M9) são baseados em técnica Fuzzy. Essa técnica foi a mais citada nos projetos de SI. O fato se justifica por ela estar associada à área de computação.

Por outro lado, seis métodos identificados na literatura são muito específicos e utilizam poucas técnicas: M4 - Dynamic Bayesian Network; M7 - Fuzzy Expert COCOMO; M11 - Importance-performance Analysis; M12 - Integrated Index; M14 - Matrix-based Risk Propagation Model; e M16 - Relative Importance Index. Os métodos M14 e M16 apresentaram apenas uma técnica de apresentação de dados cada um. Tratam-se de métodos muito específicos e que fazer referência a outros métodos com objetivo de aprimoramento.

\section{Classificação das técnicas}

A Figura 4 classifica as técnicas mais citadas na literatura para análise de risco em projetos de SI, a partir dos métodos encontrados.

Figura 4 - Classificação das técnicas por frequência de citação.

\begin{tabular}{|c|c|c|}
\hline \multicolumn{2}{|l|}{ Técnicas } & $\begin{array}{l}\text { Classificação } \\
\text { por perfil }\end{array}$ \\
\hline \multirow[t]{2}{*}{$\begin{array}{l}\text { Entrada } \\
\text { de dados }\end{array}$} & $\begin{array}{l}\text { Opinião especializada } \\
\text { Bases históricas } \\
\text { Entrevista } \\
\text { Riscos categorizados por fonte }\end{array}$ & Mais citadas \\
\hline & $\begin{array}{l}\text { Questionário } \\
\text { Delphi } \\
\text { Brainstorming } \\
\text { Observações experimentais } \\
\text { Riscos categorizados por fase }\end{array}$ & Menos citadas \\
\hline \multirow[t]{2}{*}{$\begin{array}{l}\text { Tratamento } \\
\text { de dados }\end{array}$} & $\begin{array}{l}\text { Fuzzy } \\
\text { Interdependência de riscos } \\
\text { Multicritério } \\
\text { Probabilidade e impacto } \\
\text { Interdependência de riscos } \\
\text { Multicritério }\end{array}$ & Mais citadas \\
\hline & $\begin{array}{l}\text { Probabilidade e impacto } \\
\text { Distribuição probabilística } \\
\text { Simulação }\end{array}$ & Menos citadas \\
\hline \multirow[t]{2}{*}{$\begin{array}{l}\text { Apresentação } \\
\text { de dados }\end{array}$} & $\begin{array}{l}\text { Lista priorizada de riscos } \\
\text { Rede de relacionamento }\end{array}$ & Mais citadas \\
\hline & $\begin{array}{l}\text { Matriz probabilidade-impacto } \\
\text { Tabela } \\
\text { Gráfico }\end{array}$ & Menos citadas \\
\hline
\end{tabular}


Entre as técnicas de entrada de dados mais citadas estão a opinião especializada, consulta às bases de dados históricas e entrevistas. A categorização de risco por fonte é bem citada, porém a categorização por fase de projeto e a menos citada. Pode-se inferir que em projetos de SI há uma maior preocupação com a fonte de informação em relação ao risco do que propriamente a fase do projeto.

Em relação ao tratamento de dados na análise de risco, a maioria das técnicas foram classificadas como mais citadas. Exceção a três técnicas que são baseadas em probabilidade e simulação (probabilidade e impacto, distribuição probabilística e simulação). Pode-se inferir que probabilidade e simulação não estão entre as técnicas mais utilizadas para tratamento de dados em processos de análise risco de projeto de SI. Por último cabe destacar que a técnica mais utilizada para apresentação dos dados é a lista priorizada.

\section{Conclusão}

O objetivo deste trabalho foi identificar as técnicas descritas na literatura para o gerenciamento de riscos em projetos de SI. Para isso foi realizada uma pesquisa bibliográfica nas bases de dados da Scopus, IEEE e Science Direct. A partir de 227 artigos selecionados nas bases, extraíram-se 25 artigos que foram analisados em relação aos métodos e técnicas descritas.

Entre os resultados obtidos destacam-se:

1) Métodos para análise de risco. Os métodos mais destacados na literatura para análise de risco em projetos de SI são similares àqueles frequentemente encontrados em outros campos de conhecimento, tais como Bayesian Network e Simulação de Monte Carlo. Destaca-se a associação desses métodos a uso de recursos computacionais e como consequência pode-se inferir a sua maior utilização em projetos de SI. Destaca-se também que a Simulação de Monte é um método de análise de risco amplamente referenciado pelos guias de boas práticas, tais como PMBoK, PRINCE2 e IPMA. Entretanto, o método Bayesian Network não é igualmente referenciado por esses guias de boas práticas. Há, portanto, uma oportunidade de pesquisa futura na verificação dos benefícios de uma maior adoção do método Bayesian Network na análise de risco em projetos.

2) Técnicas para análise de risco. Os resultados obtidos em relação às técnicas usadas pelos métodos de análise de risco são baseados primordialmente em técnica Fuzzy e 
multicritério. O uso de métodos baseados em Fuzzy e multicritério permite o uso de recursos computacionais. Talvez por essa razão, essas técnicas tenham sido mais citadas na literatura de SI. Da mesma forma que constatada em relação aos métodos, a técnica Fuzzy não é descrita nos guias de boas práticas mais utilizados (PMBoK, PRINCE2 e IPMA). Há também uma oportunidade de pesquisa futura na verificação dos benefícios de uma maior adoção dessa técnica na análise de risco em projetos.

O resultado mostrou uma frequência maior de trabalhos com métodos e técnicas que exigem recursos computacionais. Isso mostra que o estudo do gerenciamento de projetos em áreas de conhecimento específicas, geralmente aponta particularidades da área. $\mathrm{O}$ conhecimento dessas particularidades não só aprimora o conhecimento teórico em gerenciamento de projetos, como permite a definição de hipótese para futuras pesquisas. Dentro desse contexto, esta pesquisa contribuiu com o aumento do conhecimento sobre as características dos processos de análise de risco em projetos de SI. Contribui ainda, como uma pesquisa exploratória sobre métodos e técnicas de análise de risco, que podem servir como ponto de partida para futuras pesquisas que possam analisar quais os métodos e técnicas que melhor se aplicam ao gerenciamento de projetos em áreas e contextos específicos

\section{Referências}

Aloini, D., Dulmin, R., \& Mininno, V. (2012a). Modelling and assessing ERP project risks: A petri net approach. European Journal of Operational Research, 220(2), pp. 484-495. (2012b). Risk assessment in ERP projects. Information Systems, 37(3), pp. 183-199.

Arashpour, M. et al. (2016). Analysis of interacting uncertainties in on-site and off-site activities: Implications for hybrid construction. International Journal of Project Management, 34(7), pp. 1393-1402.

Ayala-Cruz, J. (2016). Project risk planning in high-tech new product development. Academia, 29(2), pp. 110-124.

Bardin, L. (2011). Análise de conteúdo. São Paulo: Edições 70. 
Cervo, A. L., Bervian, P. A. \& Silva, R. (2007). Metodologia científica (6a ed.). São Paulo: Pearson Prentice Hall.

Fang, C., \& Marle, F. (2013). Dealing with project complexity by matrix-based propagation modelling for project risk analysis. Journal of Engineering Design, 24(4), pp. 239-256.

Globerson, S. \& Zwikael, O. (2002). The impact of the project manager on project management planning processes. Project management journal, 33(3), pp. 58-64.

Hossen, M. M., Kang, S. \& Kim, J. (2015). Construction schedule delay risk assessment by using combined AHP-RII methodology for an international NPP project. Nuclear Engineering and Technology, 47(3), pp. 362-379.

Hu, Y. et al. (2013). Software project risk analysis using Bayesian networks with causality constraints. Decision Support Systems, 56(1), pp. 439-449.

IPMA (2015). Individual competence baseline (4 ${ }^{\text {th }}$ version). International Project Management Association.

Kitchenham, B., Brereton, O. P., Budgen, D., Turner, M., Bailey, J. \& Linkman, S. (2009). Systematic literature reviews in software engineering-a systematic literature review. Information and software technology, 51(1), pp. 7-15.

Lakatos, E. M. \& Marconi, M. de A. (2010). Fundamentos de metodologia científica. São Paulo: Atlas.

Liberzon, V. \& Archibald, R. D. (2003). From Russia with love: truly integrated project scope, schedule, resource and risk information. In: Proceedings of the 2003 PMI World Congress-Europe. [S.1.: s.n.].

López, C. \& Salmeron, J. L. (2012). Risks response strategies for supporting practitioners decision-making in software projects. In: $4^{\text {th }}$ Conference of Enterprise Information Systems - aligning technology, organizations and people (CenteriS 2012), vol. 5, pp. 437-444. 
Manalif, E. et al. (2012). Fuzzy-Excom software project risk assessment. In: Proceedings of the 11th International Conference on Machine Learning and Applications, ICMLA 2012, vol. 2, pp. 320-325.

Marle, F., Vidal, L. A. \& Bocquet, J. C. (2013). Interactions-based risk clustering methodologies and algorithms for complex project management. Anticipation of risks impacts and industrial performance evaluation in distributed organizations life cycles, 142(2), pp. 225-234.

Marmier, F., Gourc, D. \& Laarz, F. (2013). A risk oriented model to assess strategic decisions in new product development projects. Decision Support Systems, 56, pp. 74-82.

Miranda Neto, M. J. (2005). Pesquisa para o planejamento: métodos e técnicas. Rio de Janeiro: FGV.

Mohammadi, A. \& Tavakolan, M. (2013). Construction project risk assessment using combined Fuzzy and FMEA. In: Proceedings of the 2013 Joint IFSA World Congress and NAFIPS Annual Meeting, IFSA/NAFIPS 2013, pp. 232-237.

Odimabo, O. \& Oduoza, C. (2014). Methodology for project risk assessment using Bayesian belief networks in engineering construction projects. In: Proceedings of the $24^{\text {th }}$ International Conference on Flexible Automation and Intelligent Manufacturing: Capturing Competitive Advantage via Advanced Manufacturing and Enterprise Transformation - FAIM 2014, pp. 201-208.

OGC. (2009). Managing successful projects with PRINCE2. [S.1.]: The Stationery Office.

Patah, L. A. \& Carvalho, M. M. (2012). Métodos de gestão de projetos e sucesso dos projetos: um estudo quantitativo do relacionamento entre estes conceitos. Revista de Gestão e Projetos - GeP, 3(2), pp. 178-206.

PMI (2017). A Guide to the project management body of knowledge (6th edition). USA: Newtown Square, PA: Project Management Institute - PMI. 
Purnus, A. \& Bodea, C. N. (2013). Considerations on project quantitative risk analysis. In: Proceedings of the 26th International Project Management Association World Congress - IPMA, Crete, Greece, vol. 74, pp. 144-153.

Raoofpanah, H. \& Hassanlou, K. (2013). A probabilistic approach for project cost estimation using Bayesian networks. Life Science Journal, vol. 10, pp. 342-349.

Schmidt, R., Lyytinen, K. \& Keil, P. C. M. (2001). Identifying software project risks: An international Delphi study. Journal of management information systems, 17(4), pp. 536.

Standish Group (2016). Big bang boom. Inc. Boston, MA: Standish Group.

Subramanyan, H., Sawant, P. \& Bhatt, V. (2012). Construction project risk assessment: development of model based on investigation of opinion of construction project experts from India. Journal of Construction Engineering and Management, 138(3), pp. 409421.

Taylan, O. et al. (2014). Construction projects selection and risk assessment by fuzzy ANP and fuzzy TOPSIS methodologies. Applied Soft Computing, 17, pp. 105-116.

Valitov, S. \& Sirazetdinova, A. (2014). Project risks' management model on an industrial enterprise. Asian Social Science, 10(21), pp. 242-249.

Yet, B. et al. (2016). A Bayesian network framework for project cost, benefit and risk analysis with an agricultural development case study. Expert Systems with Applications, 60, pp. 141-155.

Zegordi, S., Nik, E. R. \& Nazari, A. (2012). Power plant project risk assessment using a fuzzy-ANP and fuzzy-TOPSIS method. International Journal of Engineering, Transactions B: Applications, 25(2), pp. 107-120. 